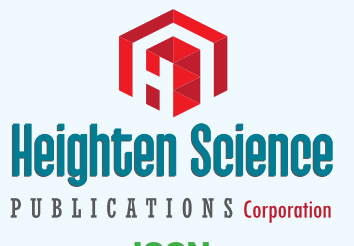

ISSN

2573-7724
Review Article

\section{Investigation of radiation attenuation parameters of some drugs used in Chemotherapy in Wide Energy Region}

\author{
Ferdi Akman ${ }^{1 *}$ and Mustafa Recep Kaçal ${ }^{2}$ \\ 'Bingöl University, Vocational School of Technical Sciences, Department of Electronic \\ Communication Technology, 12000 Bingöl, Turkey \\ ${ }^{2}$ Giresun University, Sciences and Arts Faculty, Department of Physics, 28100 Giresun, Turkey
}

*Address for Correspondence: Ferdi Akman, Bingöl University, Vocational School of

Technical Sciences, Department of Electronic Communication Technology, 12000 Bingöl, Turkey, Tel: +904262160012-4067; Fax: +904262150788; Email: fakman@bingol.edu.tr

Submitted: 20 July 2018

Approved: 03 August 2018

Published: 04 August 2018

Copyright: @ 2018 Akman F, et al. This is an open access article distributed under the Creative Commons Attribution License, which permits unrestricted use, distribution, and reproduction in any medium, provided the original work is properly cited.

Keywords: Chemotherapy drug; Mass attenuation Coefficient; Linear attenuation Coefficient; Half value layer; Mean free path; Effective atomic number

\section{Abstract}

Objectives: The aim of this study is to compute the radiation attenuation parameters such as mass attenuation coefficient, linear attenuation coefficient, half value layer, mean free path, and effective atomic number for some selected chemotherapy drugs such as Lomustine, Cisplatin, Carmustine, and Chlorambucil in the energy range from $1 \mathrm{keV}$ to $100 \mathrm{GeV}$.

Materials and Methods: The mass attenuation coefficients were calculated with the help of WinXCOM program. Using the obtained mass attenuation coefficients, other parameters such as linear attenuation coefficient, half value layer, mean free path, and effective atomic number were derived.

Results: It is observed that the variations of these parameters with respect to the photon energy show changes in different energy regions. According to the obtained results, Cisplatin has the highest mass attenuation coefficient, linear attenuation coefficient and effective atomic number results among the selected chemotherapy drugs. Moreover, Chlorambucil has the highest half value layer and mean free path results among the selected chemotherapy drugs.

Conclusions: The results of this study are useful for applied science fields such as radiation physics, pharmacology, and medical physics. These results may be useful when the selected chemotherapy drugs are used together with radiology.

\title{
Introduction
}

Ionizing radiation is frequently used in medicine for diagnostic and therapeutic. Therefore, the accurate values of attenuation parameters for this type radiation are very important. Lomustine, Cisplatin, Carmustine, and Chlorambucil are very wellknown chemotherapeutic drugs. These chemotherapy drugs are class of alkylating agents which are a class of antineoplastic or anticancer drugs. The drugs in this class inhibit the transcription of DNA into RNA and thus stop the protein synthesis [1]. Carmustine and Lomustine is utilized alone or combined with other drugs to treat various types' cancer such as testicular, pancreatic, ovarian, lymphoma and brain. Chlorambucil is usually preferred in the treatment of chronic lymphocytic leukemia, Hodgkin's and non-Hodgkin's lymphomas [2]. Cisplatin is usually chosen because of its strong antitumor activity, but its side effects contain renal toxicity, nausea and vomiting. Cisplatin is generally preferred in the treatment of chemotherapy for lung, ovarian, breast, and brain cancers [3]. Furthermore, radioactive Cisplatin with low doses and short half-life is used in such treatment [4]. The information on radiation attenuation parameters may be useful when these drugs are used together with radiology. The parameters such as mass attenuation coefficient, linear attenuation coefficient, half value layer, tenth value layer, mean free path, effective atomic number, and effective electron density are quantities to provide information about the radiation attenuation 
in case of interaction of matter with radiation. These parameters are important for many applied sciences such as health physics, engineering, radiation physics, and space research. There are many scientific studies carried out for these parameters using the different type matters in the literature [5-15]. The aim of this study is to investigate the radiation attenuation parameters such as mass attenuation coefficient, linear attenuation coefficient, half value layer, mean free path, and effective atomic number of commonly used in chemotherapeutic drugs such as Lomustine, Cisplatin, Carmustine, and Chlorambucil. The calculations were carried out with the aid of the WinXCOM program [16] in the energy range from $1 \mathrm{keV}$ to $100 \mathrm{GeV}$. The obtained theoretical results are shown graphically and interpreted for different interaction processes, namely, photoelectric absorption, Compton scattering, and pair production.

\section{Theoretical calculation process}

The mass attenuation coefficient of any matter having more than one element can be computed by the following equation;

$$
(\mu / \rho)_{\text {matter }}=\sum_{i=1} W_{i}(\mu / \rho)_{i}
$$

In the equation, $\mathrm{W}_{\mathrm{i}}$ and $(\mu / \rho)$ imply the weight fraction and mass attenuation coefficient of related element in the matter, respectively. This equation is also known as mixture rule. According to the rule, the mass attenuation coefficient of matter is the sum of the mass attenuation coefficients contributed by the weight fraction of each element in the matter. In the present study, these calculations were achieved with the help of the WinXCOM program. As is well-known, the mass attenuation coefficient depends on the phase state of the matter. Another parameter independent of the phase state of matter and associated with the mass attenuation coefficient is the linear attenuation coefficient. It can be obtained from the mathematical multiplication of the mass attenuation coefficient and density of the matter. Other two radiation attenuation parameters that can be derived using the linear attenuation coefficient are half value layer and mean free path. The half value layer is defined as the thickness required to decrease the radiation intensity by one-half. The mean free path is known as the thickness a photon travels before interacting. The mathematical relationships of these two parameters to the linear attenuation coefficient are given in the following equations;

$$
\begin{aligned}
& H V L=\frac{\ln 2}{\mu}=\frac{0.693}{\mu}(2) \\
& M F P=\frac{1}{\mu} \text { (3) }
\end{aligned}
$$

Another radiation attenuation parameter, which is important in matters with more than one element, is the effective atomic number. This parameter can be computed using the mass attenuation coefficient as given in the following equation;

$$
Z_{e f f}=\frac{\sum_{i} f_{i} A_{i}(\mu / \rho)_{i}}{\sum_{j} f_{j} \frac{A_{j}}{Z_{j}}(\mu / \rho)_{j}}
$$

In the equation, $\mathrm{A}_{\mathrm{i}}$ implies the atomic weight, $\mathrm{Z}_{\mathrm{i}}$ represents the atomic number and $\mathrm{f}_{\mathrm{i}}$ implies the fractional abundance of the related element in the matter.

\section{Results and Discussion}

The chemical formulas, physical densities and molecular weights of Camustine, Chlorambucil, Lomustine, and Cisplatin drugs are given in Table 1. The mass attenuation coefficients, linear attenuation coefficients, half value layers, mean free paths, and 
effective atomic numbers of the selected drugs were computed with the help of the information given in Table 1 . The determined mass attenuation coefficients by the WinXCOM program are graphically demonstrated in Figure 1. The linear attenuation coefficient and effective atomic number results, which are directly dependent on the mass attenuation coefficient, against energy are presented in Figures 2-3, respectively. As clearly seen from Figures 1-3, it is observed that the results of these parameters show different changes in different energy regions. For this reason, the interpretation of these figures should be made according to the different interaction processes, namely, they should be interpreted with photoelectric absorption, Compton scattering and pair production processes. In the low energy region, it is observed that the mass attenuation coefficient, linear attenuation coefficient and effective atomic number values decrease sharply and exponentially with respect to the energy. The reason for this observation is the photoelectric absorption which is predominant interaction process in the low energy region. The proportions between photoelectric absorption and energy, and atomic number are $1 / \mathrm{E}^{3.5}$ and $\mathrm{Z}^{4-5}$, respectively. In the medium energy region, it is seen that the variations in these parameters against the energy are slighter, or even almost constant. The dominant interaction in this region is Compton scattering and it is inversely proportional to energy (E), and directly proportional to the atomic number (Z).

Table 1: The chemical formulas, physical densities and molecular weights of the selected drug samples.

\begin{tabular}{|c|c|c|c|}
\hline Sample Name & Chemical structure & Density $\left(\mathrm{g} / \mathrm{cm}^{3}\right)$ & Molecular weight $(\mathrm{g} / \mathrm{mol})$ \\
\hline Carmustine & $\mathrm{C}_{5} \mathrm{H}_{9} \mathrm{Cl}_{2} \mathrm{~N}_{3} \mathrm{O}_{2}$ & 1.46 & 214.05 \\
\hline Chlorambucil & $\mathrm{C}_{14} \mathrm{H}_{19} \mathrm{Cl}_{2} \mathrm{NO}_{2}$ & 1.25 & 304.21 \\
\hline Lomustine & $\mathrm{C}_{9} \mathrm{H}_{16} \mathrm{CIN}_{3} \mathrm{O}_{2}$ & 1.35 & 233.69 \\
\hline Cisplatin & $\mathrm{Pt}\left(\mathrm{NH}_{3}\right)_{2} \mathrm{Cl}_{2}$ & 3.70 & 300.04 \\
\hline
\end{tabular}

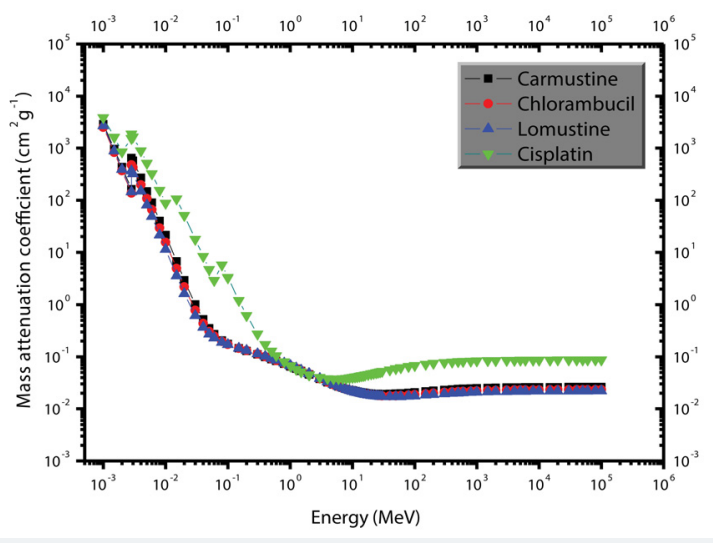

Figure 1: The graph of mass attenuation coefficient versus photon energy for the selected chemotherapy drugs.

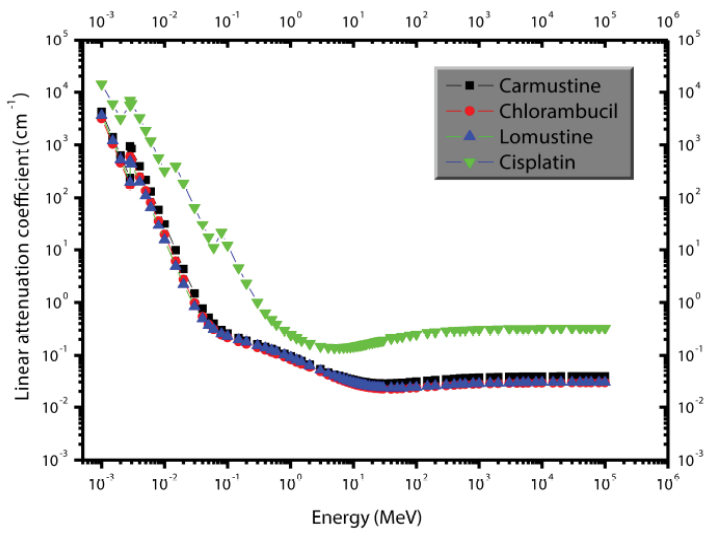

Figure 2: The graph of linear attenuation coefficient versus photon energy for the selected chemotherapy drugs. 


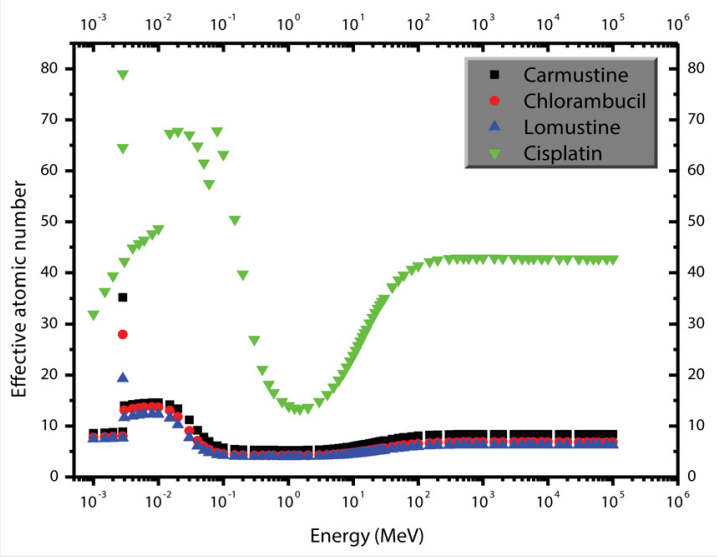

Figure 3: The variation of effective atomic number against photon energy for the selected chemotherapy drugs.

It is observed that these parameters increase again in the high energy region. The dominant interaction in this region is pair production which is directly proportional with the energy and atomic number as $\mathrm{E}$ and $\mathrm{Z}^{2}$, respectively. Similar observations have been reported by Kavaz et al. [17] who calculated the some attenuation parameters such as energy absorption buildup factor, exposure buildup factor, effective atomic number and effective electron density for some selected chemotherapy drugs. According to the Figures 1-2, Cisplatin has the highest mass and linear attenuation coefficients among the selected drug samples due to the presence of the platinum element. This element has the largest atomic number in all selected drug samples. We can say that the mass attenuation coefficient increases with increasing atomic number. Moreover, the linear attenuation coefficient increases with increasing density (Table 1). The half value layer and mean free path results of selected chemotherapy drugs, derived using the linear attenuation coefficients, are illustrated in Figures 4-5, respectively. In the low energy region, the half value layers and mean free paths are almost constant and then they increase sharply with respect to the energy. In the medium energy region, they have the highest values. In the last energy region, namely, in the high energy region, the half value layer and mean free path results against energy decrease exponentially. So, the exact opposite observations in mass and linear attenuation coefficients is observed in the half value layers and mean free paths. The reason for these observations that as can be easily seen from equations $2-3$, the linear attenuation coefficient is inversely proportional to half value layer and mean free path. The highest half value layer and mean free path values were found for Chlorambucil drug, while the lowest half value layer and mean free path values were found for Cisplatin drug among the selected chemotherapy drugs. This means that a thinner Cisplatin drug will be sufficient to absorb any given energy, while a thicker Chlorambucil drug will be needed.

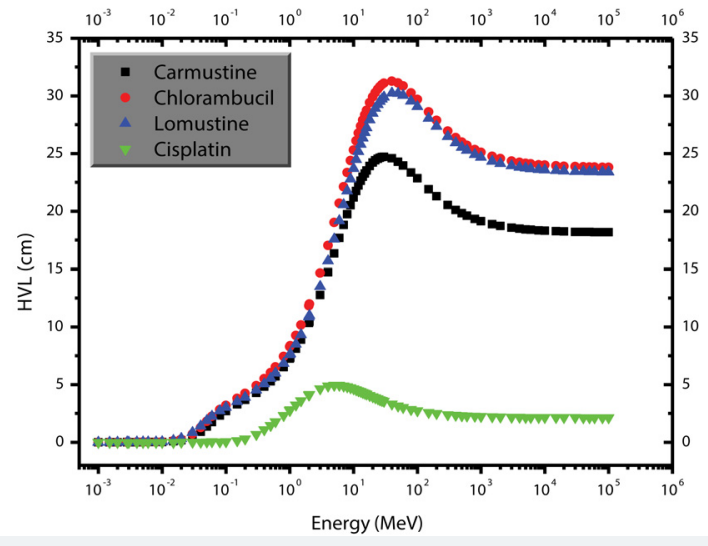

Figure 4: The change of half value layer versus photon energy for the selected chemotherapy drugs. 


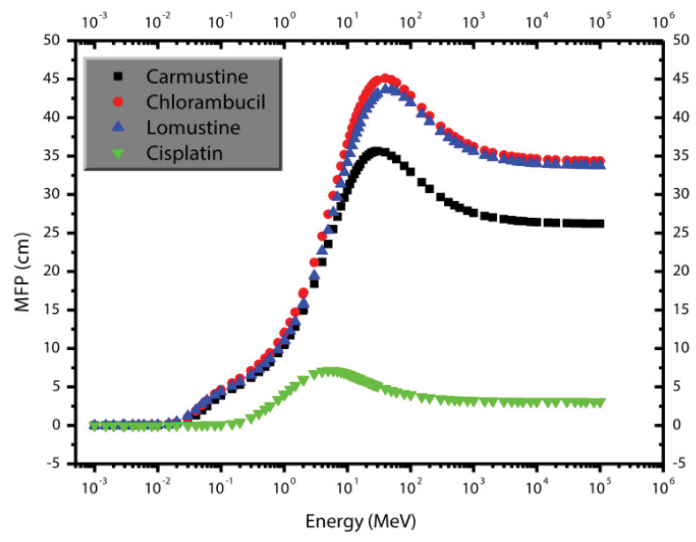

Figure 5: The change of mean free path versus photon energy for the selected chemotherapy drugs.

\section{Conclusion}

In the present research, the some radiation attenuation parameters such as mass attenuation coefficient, linear attenuation coefficient, half value layer, mean free path, and effective atomic number of selected chemotherapy drugs such as Carmustine, Chlorambucil, Lomustine, and Cisplatin were investigated in wide energy region. It was observed that these parameter change depending on different interaction processes. The highest values of mass attenuation coefficient, linear attenuation coefficient, and effective atomic number were observed in the low energy region and the highest values of half value layer, and mean free path were observed in the medium energy region. Cisplatin is the chemotherapy drug having the best radiation attenuation characteristics among the selected drug samples. The results of this study are useful for applied science fields such as radiation physics, pharmacology, and medical physics. Besides, the presented results may be useful when the selected chemotherapy drugs are used together with radiology.

\section{References}

1. National Institutes of Health, 2012.

2. Weiss RB, Issell BF. The nitrosoureas: carmustine (BCNU) and lomustine (CCNU). Cancer Treat Rev. 1982; 9: 313-330. Ref.: https://tinyurl.com/y8qdebfs

3. Dasari S, Tchounwou PB. Cisplatin in cancer therapy: molecular mechanisms of action. Eur $J$ Pharmacol. 2014; 740: 364-378. Ref.: https://tinyurl.com/ycxfuo7r

4. Bodnar EN, Dikiy MP, Medvedeva EP. Photonuclear production and antitumor effect of radioactive cisplatin (195mPt). J Radionan Nucl Ch. 2015; 305: 133-138. Ref.: https://tinyurl.com/ycwcen5h

5. Akman F, Durak R, Kacal MR, Bezgin F. Study of absorption parameters around the $\mathrm{K}$ edge for selected compounds of Gd. X-ray Spectrom. 2016; 45: 103-110. Ref.: https://tinyurl.com/yapxhshz

6. Akman F, Durak R, Turhan MF, Kacal MR. Studies on effective atomic numbers, electron densities from mass attenuation coefficients near the $\mathrm{K}$ edge in some samarium compounds. Appl Radiat Isotopes. 2015; 101: 107-113. Ref.: https://tinyurl.com/yba3c4bx

7. Sayyed MI. Half value layer, mean free path and exposure buildup factor for tellurite glasses with different oxide compositions. J Alloy Compd. 2017; 695: 3191-3197. Ref.: https://tinyurl.com/yaehr6zn

8. Akman F, Durak R, Kacal MR. Determination of $K$ shell absorption parameters for some lanthanides using the X-ray attenuation method. Can J Phys. 2015; 93: 1532-1540. Ref.: https://tinyurl.com/yctgdyjp

9. Kaçal MR, Karataş HA, Akman F. Photon absorption characteristics of some selected enzyme inhibitors used in cancer research in the energy range $1 \mathrm{keV}-100 \mathrm{GeV}$. J Radiol Oncol. 2017; 1: 60-68. Ref.: https://tinyurl.com/yd36ejhb

10. Akman F, Kaçal MR, Akman F, Soylu MS. Determination of effective atomic numbers and electron densities from mass attenuation coefficients for some selected complexes containing lanthanides. Can J Phys. 2017; 95: 1005-1011. Ref.: https://tinyurl.com/y9y32rjm 
11. Akman $F$, Kaçal MR, Durak R. Chemical effect on the $K$ shell absorption parameters of some selected cerium compounds. J Instrum. 2016; 11: P08006. Ref.: https://tinyurl.com/ybmuet9x

12. Kacal MR, Han I, Akman F. Measurement of mass attenuation coefficients by Si ( $\mathrm{Li}), \mathrm{Nal}(\mathrm{TI})$, and Cd (TI) detectors. Nuclear Science and Technology. 2012: 59-69. Ref.: https://tinyurl.com/y7u2qgx2

13. Sayyed MI, Issa SAM, Auda SH. Assessment of radio-protective properties of some anti-inflammatory drugs. Prog Nucl Energ. 2017; 100: 297-308. Ref.: https://tinyurl.com/ycfc6s9y

14. Akman F, Geçibesler IH, Sayyed MI, Tijani SA, Tufekci AR, et al. Determination of some useful radiation interaction parameters for waste foods. Nucl Eng Technol. 2018; 50: 944-949. Ref.: https://tinyurl.com/ybtqtw2z

15. Akman F, Geçibesler iH, Demirkol I, Çetin A. Determination of effective atomic numbers and electron densities for some synthesized triazoles from the measured total mass attenuation coefficients at different energies. Can J Phys. Ref.: https://tinyurl.com/y7w8sgny

16. Gerward L, Guilbert N, Jensen KB, Levring H. X-ray absorption in matter: Reengineering XCOM. Radiat Phys Chem. 2001; 60: 23-24. Ref.: https://tinyurl.com/y8v5yhz3

17. Kavaz E, Ahmadishadbad N, Özdemir Y. Photon buildup factors of some chemotherapy drugs. Biomed Pharmacother. 2015; 69: 34-41. Ref.: https://tinyurl.com/y7858kgj 